\title{
ERRATUM
}

\section{Growth hormone upregulates the pro-tumorigenic galectin 1 in mouse liver}

\author{
María L Bacigalupo ${ }^{1, *}$, Verónica G Piazza ${ }^{1, *}$, Nadia S Cicconi' ${ }^{1}$, Pablo Carabias ${ }^{1}$, Andrzej Bartke², Yimin Fang², \\ Ana I Sotelo ${ }^{1}$, Gabriel A Rabinovich ${ }^{3}$, María F Troncoso ${ }^{1, \dagger}$ and Johanna G Miquet ${ }^{1, \dagger}$ \\ 1'Departamento de Química Biológica, Facultad de Farmacia y Bioquímica, Universidad de Buenos Aires, Consejo Nacional de Investigaciones Científicas y \\ Técnicas, Instituto de Química y Fisicoquímica Biológicas, Buenos Aires, Argentina \\ ${ }^{2}$ Department of Internal Medicine, Geriatrics Research, Southern Illinois University School of Medicine, Springfield, Illinois, USA \\ ${ }^{3}$ Laboratorio de Inmunopatología, Instituto de Biología y Medicina Experimental, Consejo Nacional de Investigaciones Científicas y Técnicas, and \\ Departamento de Química Biológica, Facultad de Ciencias Exactas y Naturales, Universidad de Buenos Aires, Buenos Aires, Argentina
}

Correspondence should be addressed to M F Troncoso or J G Miquet: fernanda@qb.ffyb.uba.ar or jmiquet@qb.ffyb.uba.ar

The authors and journal apologise for an error in the above paper, which appeared in volume 8 part 8, pages 1108-1117. The error relates to the Cyclophilin A R primer given on page 1110.

The original article stated:

'Cyclophilin A ... R: TCAGCCTGGTCAAAGGTGAT'

This should have stated:

'Cyclophilin A ... R: AAGTCACCACCCTGGCAC'

(c) 2021 The authors Published by Bioscientifica Ltd 